\title{
Detecting Distributed Denial-of-Service DDoS Attacks
}

\author{
Fasidi $\mathrm{FO}^{1}$ and Adebayo $\mathrm{OT}^{2 *}$ \\ ${ }^{1}$ Department of Computer Science, Federal University of Technology, Akure, Nigeria \\ ${ }^{2}$ Department of Information Technology, Federal University of Technology, Nigeria
}

*Corresponding author: Adebayo OT, Department of Information Technology, Federal University of Technology, Akure, Nigeria

\begin{abstract}
Since the number of damage cases resulting from distributed denial-of-service (DDoS) attacks has recently been increasing, the need for agile detection and appropriate response mechanisms against DDoS attacks has also been increasing. The latest DDoS attack has the property of swift propagation speed and various attack patterns. There is therefore a need to create a lighter mechanism to detect and respond to such new and transformed types of attacks with greater speed. In a wireless network system, the security is a main concern for a user.
\end{abstract}

\section{Introduction}

Security of information is of utmost importance to organization striving to survive in a competitive marketplace. Network security has been an issue since computer networks became prevalent, most especially now that internet is changing the face of computing. As dependency on Internet increases on daily basis for business transaction, so also is cyber-attacks by intruder who exploit flaws in Internet architecture, protocol, operating systems and application software to carry out their nefarious activities Such hosts can be compromised within a short time to run arbitrary and potentially malicious attack code transported in a worm or virus or injected through installed backdoors. Distributed denial of service (DDoS) use such poorly secured hosts as attack platform and cause degradation and interruption of Internet services, which result in major financial losses, especially if commercial servers are affected (Duberdorfer, 2004).

\section{Related Works}

Brignoli et al. [1] proposed DDoS detection based on traffic selfsimilarity estimation, this approach is a relatively new approach which is built on the notion that undisturbed network traffic displays fractal like properties. These fractal-like properties are known to degrade in presence of abnormal traffic conditions like DDoS. Detection is possible by observing the changes in the level of self-similarity in the traffic flow at the target of the attack. Existing literature assumes that DDoS traffic lacks the self-similar properties of undisturbed traffic. The researcher shows how existing bot- nets could be used to generate a self-similar traffic flow and thus break such assumptions. Streilien et al,2005. Worked on detection of DoS attacks through the polling of Remote Monitoring (RMON) capable devices. The researchers developed a detection algorithm for simulated flood-based DoS attacks that achieves a high detection rate and low false alarm rate.

Yeonhee Lee [2] focused on a scalability issue of the anomaly detection and introduced a Hadoop based DDoS detection scheme to detect multiple attacks from a huge volume of traffic. Different from other single host-based approaches trying to enhance memory efficiency or to customize process complexity, our method leverages Hadoop to solve the scalability issue by parallel data processing. From experiments, we show that a simple counterbased DDoS attack detection method could be easily implemented in Hadoop and shows its performance gain of using multiple nodes in parallel. It is expected that a signature-based approach could be well suited with Hadoop. However, we need to tackle a problem to develop a real time defense system, because the current Hadoop is oriented to batch processing.

\section{Proposed System Architecture of Intrusion Detection Based on Association Rule}

The structure of the proposed architecture for real time detection of Dos instruction detection via association rule mining, 
it is divided into two phases: learning and testing. The network sniffer processed the tcpdump binary into standard format putting into learning, during the learning phase, duplicate records as well as columns with single same data were expunge from the record so as to reduce operational. Another table Hashmap was created by the classification model to keep track of the count of various likely classmark that can match the current read network traffic, this table will be discarded once the classmark with highest count had been selected. Depicted in Table 1 is the Association rule classifier algorithm (Tables 2-4).

Table 1: Association Rule Mining Classifier Algorithm.

1.Read the first traffic data from table 3.4 into the memory

2.Read the first rule from table 3.2

3.Read the first number table 3.3

4.Check the corresponding attribute(s) of the number read in table 3.4

5.Check if the attribute matches the rule read

6.If it matches, make an entry/increment of the attack-type in the rule read in the hash

map, and go to (3) to read the next number

7.Repeat (4) to (6) again until all the number had been read

8.Read the next rule in (2) and perform (3) to (7) until all the rules had been read.

9.Check the attack entry with highest count in the hash map table,and assign the attack

Table 2: Sample Rules.

\begin{tabular}{|c|c|}
\hline Neptune: CONFIDENCE $=100.0 \%$, SUPP & $1926388830027008 \%$ \\
\hline $\begin{array}{l}\text { private->teardrop: } \\
\text { SUPPORT }=0.012714166278178252 \%\end{array}$ & CONFIDENCE $=100.0 \%$, \\
\hline $\begin{array}{l}\text { finger->land: } \\
\text { SUPPORT }=1.2842592200180053 \mathrm{E}-4 \%\end{array}$ & CONFIDENCE $=100.0 \%$, \\
\hline 1032->smurf:CONFIDENCE $=100.0 \%, S U$ & $4.7445672624345185 \%$ \\
\hline $\begin{array}{l}\text { 0.12->land: CONFIDENCE }=100.0 \%, \text { SU } \\
\text { 4\%cp, } \quad \text { telnet->Neptune: } \\
\text { SUPPORT }=0.001926388830027008 \%\end{array}$ & $\begin{array}{l}1.2842592200180053 E- \\
\text { ENCE }=\quad 100.0 \%,\end{array}$ \\
\hline $\begin{array}{l}\text { tcp, } \quad \text { finger }->\text { land: } \\
\text { SUPPORT=1.2842592200180053E-4\% }\end{array}$ & CONFIDENCE $=100.0 \%$, \\
\hline $\begin{array}{l}\text { udp, } \quad \text { private->teardrop: } \\
\text { SUPPORT=0.012714166278178252\% }\end{array}$ & CONFIDENCE $=100.0 \%$, \\
\hline $\begin{array}{l}\text { udp, } \quad \text { SF->teardrop: } \\
\text { SUPPORT }=0.012714166278178252 \%\end{array}$ & CONFIDENCE $=100.0 \%$, \\
\hline
\end{tabular}

\begin{tabular}{|c|c|}
\hline $\begin{array}{l}\text { icmp,1032-> } \quad \text { smurf: } \\
\text { SUPPORT }=4.7445672624345185 \%\end{array}$ & CONFIDENCE $=100.0 \%$, \\
\hline $\begin{array}{l}\text { icmp,1480->pod: } \\
\text { SUPPORT=0.0025685184400360108\% }\end{array}$ & CONFIDENCE $=100.0 \%$, \\
\hline $\begin{array}{l}\text { udp,28->teardrop: } \\
\text { SUPPORT=0.012714166278178252\% }\end{array}$ & CONFIDENCE $=100.0 \%$, \\
\hline $\begin{array}{l}\text { tcp,1->land: } \\
\text { SUPPORT=1.2842592200180053E-4\% }\end{array}$ & CONFIDENCE $=100.0 \%$, \\
\hline $\begin{array}{l}\text { icmp,0->smurf: } \\
\text { SUPPORT=4.7445672624345185\% }\end{array}$ & CONFIDENCE $=100.0 \%$, \\
\hline $\begin{array}{l}\text { icmp,1->pod: } \\
\text { SUPPORT=0.0025685184400360108\% }\end{array}$ & CONFIDENCE $=100.0 \%$, \\
\hline $\begin{array}{l}\text { udp,3->teardrop: } \quad \text { CONFI } \\
\text { SUPPORT }=0.012585740356176451 \%\end{array}$ & 8.98989898989899\%, \\
\hline $\begin{array}{l}\text { icmp,511->smurf: } \quad \text { CONFI } \\
\text { SUPPORT }=4.417081161329928 \%\end{array}$ & $93.09766132524902 \%$ \\
\hline $\begin{array}{l}\text { icmp,255->smurf: } \quad \text { CONFI } \\
\text { SUPPORT }=4.730568836936323 \%\end{array}$ & $99.70495885664789 \%$, \\
\hline $\begin{array}{l}\text { tcp, } \\
\text { SUPPORT=1.2842592200180053E-4\% }\end{array}$ & CONFIDENCE $=100.0 \%$, \\
\hline $\begin{array}{l}\text { icmp,1.00->smurf: } \quad \text { CONFI } \\
\text { SUPPORT }=4.712075504168063 \%\end{array}$ & $99.31517973148549 \%$, \\
\hline $\begin{array}{l}\text { icmp,0.04->pod: } \\
\text { SUPPORT }=0.0020548147520288084 \%\end{array}$ & CONFIDENCE $=80.0 \%$, \\
\hline
\end{tabular}

Table 3: Sampled number combination table.

\begin{tabular}{|c|c|c|c|}
\hline & $0,1,20$ & $1,3,20$ & $2,6,20$ \\
\hline 0,20 & $0,2,20$ & $1,4,20$ & $2,7,20$ \\
\hline 1,20 & $0,3,20$ & $1,5,20$ & $2,8,20$ \\
\hline 2,20 & 0.4 .20 & 16.20 & 2920 \\
\hline 3,20 & $0,1,20$ & $1,0,20$ & \\
\hline 4,20 & $0,5,20$ & $1,7,20$ & $2,10,20$ \\
\hline 5.20 & $0,6,20$ & $1,8,20$ & $2,11,20$ \\
\hline 620 & $0,7,20$ & $1,9,20$ & $2,12,20$ \\
\hline 720 & $0,8,20$ & $1,10,20$ & $2,13,20$ \\
\hline 820 & $0,9,20$ & $1,11,20$ & $2,14,20$ \\
\hline & $0,10,20$ & $1,12,20$ & $2,15,20$ \\
\hline 1020 & $0,11,20$ & $1,13,20$ & $2,16,20$ \\
\hline 11,20 & $0,12,20$ & $1,14,20$ & $2,17,20$ \\
\hline 12,20 & $0,13,20$ & $1,15,20$ & $2,18,20$ \\
\hline 13,20 & $0,14,20$ & $1,16,20$ & $2,19,20$ \\
\hline 14.20 & $0,15,20$ & $1,17,20$ & $3,4,20$ \\
\hline 15,20 & $0,16,20$ & $1,18,20$ & $3,5,20$ \\
\hline 1620 & $0,17,20$ & $1,19,20$ & $3,6,20$ \\
\hline 1720 & $0,18,20$ & $2,3,20$ & $3,7,20$ \\
\hline & $0,19,20$ & $2,4,20$ & $3,8,20$ \\
\hline 10,20 & $1,2,20$ & $2,5,20$ & $3,9,20$ \\
\hline
\end{tabular}


Table 4: Sample Network Traffic Data.

\begin{tabular}{|c|c|c|c|c|c|c|c|c|c|c|c|c|c|c|c|c|c|c|c|}
\hline tcp & telnet & S0 & 0 & 0 & 0 & 2 & 1 & 0.5 & 1 & 0.5 & 1 & 1 & 2 & 1 & 0 & 1 & 1 & 1 & 0.5 \\
\hline tcp & telnet & So & 0 & 0 & 0 & 3 & 2 & 0.67 & 1 & 0.67 & 0.67 & 2 & 3 & 1 & 0 & 0.5 & 0.67 & 1 & 0.67 \\
\hline tcp & telnet & So & 0 & 0 & 0 & 4 & 3 & 0.75 & 1 & 0.75 & 0.5 & 3 & 4 & 1 & 0 & 0.33 & 0.5 & 1 & 0.75 \\
\hline tcp & telnet & So & 0 & 0 & 0 & 5 & 4 & 0.8 & 1 & 0.8 & 0.4 & 4 & 5 & 1 & 0 & 0.25 & 0.4 & 1 & 0.8 \\
\hline tcp & telnet & So & 0 & 0 & 0 & 6 & 5 & 0.83 & 1 & 0.83 & 0.33 & 5 & 6 & 1 & 0 & 0.2 & 0.33 & 1 & 0.83 \\
\hline icmp & ecr_i & SF & 1032 & 0 & 0 & 511 & 511 & 0 & 0 & 1 & 0 & 196 & 51 & 0.26 & 0.02 & 0.26 & 0 & 0 & 0 \\
\hline icmp & ecr_i & SF & 1032 & 0 & 0 & 511 & 511 & 0 & 0 & 1 & 0 & 197 & 52 & 0.26 & 0.02 & 0.26 & 0 & 0 & 0 \\
\hline icmp & ecr_i & SF & 1032 & 0 & 0 & 511 & 511 & 0 & 0 & 1 & 0 & 198 & 53 & 0.27 & 0.02 & 0.27 & 0 & 0 & 0 \\
\hline icmp & ecr_i & SF & 1032 & 0 & 0 & 511 & 511 & 0 & 0 & 1 & 0 & 199 & 54 & 0.27 & 0.02 & 0.27 & 0 & 0 & 0 \\
\hline icmp & ecr_i & SF & 1032 & 0 & 0 & 511 & 511 & 0 & 0 & 1 & 0 & 200 & 55 & 0.28 & 0.01 & 0.28 & 0 & 0 & 0 \\
\hline icmp & ecr_i & SF & 1032 & 0 & 0 & 511 & 511 & 0 & 0 & 1 & 0 & 201 & 56 & 0.28 & 0.01 & 0.28 & 0 & 0 & 0 \\
\hline icmp & ecr_i & SF & 1032 & 0 & 0 & 511 & 511 & 0 & 0 & 1 & 0 & 202 & 57 & 0.28 & 0.01 & 0.28 & 0 & 0 & 0 \\
\hline icmp & ecr_i & SF & 1032 & 0 & 0 & 511 & 511 & 0 & 0 & 1 & 0 & 203 & 58 & 0.29 & 0.01 & 0.29 & 0 & 0 & 0 \\
\hline icmp & ecr_i & SF & 1032 & 0 & 0 & 511 & 511 & 0 & 0 & 1 & 0 & 204 & 59 & 0.29 & 0.01 & 0.29 & 0 & 0 & 0 \\
\hline icmp & ecr_i & SF & 1032 & 0 & 0 & 511 & 511 & 0 & 0 & 1 & 0 & 205 & 60 & 0.29 & 0.01 & 0.29 & 0 & 0 & 0 \\
\hline icmp & ecr_i & SF & 1032 & 0 & 0 & 511 & 511 & 0 & 0 & 1 & 0 & 206 & 61 & 0.3 & 0.01 & 0.3 & 0 & 0 & 0 \\
\hline icmp & ecr_i & SF & 1032 & 0 & 0 & 511 & 511 & 0 & 0 & 1 & 0 & 207 & 62 & 0.3 & 0.01 & 0.3 & 0 & 0 & 0 \\
\hline icmp & ecr_i & SF & 1032 & 0 & 0 & 511 & 511 & 0 & 0 & 1 & 0 & 208 & 63 & 0.3 & 0.01 & 0.3 & 0 & 0 & 0 \\
\hline icmp & ecr_i & SF & 1032 & 0 & 0 & 511 & 511 & 0 & 0 & 1 & 0 & 209 & 64 & 0.31 & 0.01 & 0.31 & 0 & 0 & 0 \\
\hline icmp & ecr_i & SF & 1032 & 0 & 0 & 511 & 511 & 0 & 0 & 1 & 0 & 210 & 65 & 0.31 & 0.01 & 0.31 & 0 & 0 & 0 \\
\hline icmp & ecr_i & SF & 1032 & 0 & 0 & 511 & 511 & 0 & 0 & 1 & 0 & 211 & 66 & 0.31 & 0.01 & 0.31 & 0 & 0 & 0 \\
\hline icmp & ecr_i & SF & 1032 & 0 & 0 & 511 & 511 & 0 & 0 & 1 & 0 & 212 & 67 & 0.32 & 0.01 & 0.32 & 0 & 0 & 0 \\
\hline icmp & ecr_i & SF & 1032 & 0 & 0 & 511 & 511 & 0 & 0 & 1 & 0 & 213 & 68 & 0.32 & 0.01 & 0.32 & 0 & 0 & 0 \\
\hline icmp & ecr_i $\mathrm{i}$ & SF & 1032 & 0 & 0 & 511 & 511 & 0 & 0 & 1 & 0 & 214 & 69 & 0.32 & 0.01 & 0.32 & 0 & 0 & 0 \\
\hline icmp & ecr_i & $\mathrm{SF}$ & 1032 & 0 & 0 & 511 & 511 & 0 & 0 & 1 & 0 & 215 & 70 & 0.33 & 0.01 & 0.33 & 0 & 0 & 0 \\
\hline icmp & ecr_i $\mathrm{i}$ & SF & 1032 & 0 & 0 & 511 & 511 & 0 & 0 & 1 & 0 & 216 & 71 & 0.33 & 0.01 & 0.33 & 0 & 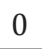 & 0 \\
\hline icmp & ecr_i & SF & 1032 & 0 & 0 & 511 & 511 & 0 & 0 & 1 & 0 & 217 & 72 & 0.33 & 0.01 & 0.33 & 0 & U & 0 \\
\hline icmp & ecr_i & SF & 1032 & 0 & 0 & 509 & 509 & 0 & 0 & 1 & 0 & 218 & 73 & 0.33 & 0.01 & 0.33 & 0 & 0 & 0 \\
\hline icmp & ecr_i $\mathrm{i}$ & SF & 1032 & 0 & 0 & 2 & 2 & 0 & 0 & 1 & 0 & 219 & 74 & 0.34 & 0.01 & 0.34 & 0 & 0 & 0 \\
\hline icmp & ecr_i & SF & 1032 & 0 & 0 & 317 & 317 & 0 & 0 & 1 & 0 & 220 & 75 & 0.34 & 0.01 & 0.34 & 0 & 0 & 0 \\
\hline icmp & ecr_i & SF & 1032 & 0 & 0 & 318 & 318 & 0 & 0 & 1 & 0 & 221 & 76 & 0.34 & 0.01 & 0.34 & 0 & 0 & 0 \\
\hline icmp & ecr_i $\mathrm{i}$ & SF & 1032 & 0 & 0 & 511 & 511 & 0 & 0 & 1 & 0 & 222 & 77 & 0.35 & 0.01 & 0.35 & 0 & 0 & 0 \\
\hline icmp & ecr_i & SF & 1032 & 0 & 0 & 511 & 511 & 0 & 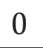 & 1 & 0 & 223 & 78 & 0.35 & 0.01 & 0.35 & 0 & 0 & 0 \\
\hline icmp & ecr_i & SF & 1032 & 0 & 0 & 511 & 511 & 0 & 0 & 1 & 0 & 224 & 79 & 0.35 & 0.01 & 0.35 & 0 & 0 & 0 \\
\hline icmp & ecr_i & SF & 1032 & 0 & 0 & 511 & 511 & 0 & 0 & 1 & 0 & 225 & 80 & 0.36 & 0.01 & 0.36 & 0 & 0 & 0 \\
\hline icmp & ecr_i & SF & 1032 & 0 & 0 & 511 & 511 & 0 & 0 & 1 & 0 & 226 & 81 & 0.36 & 0.01 & 0.36 & 0 & 0 & 0 \\
\hline icmp & ecr_i & SF & 1032 & 0 & 0 & 511 & 511 & 0 & 0 & 1 & 0 & 227 & 82 & 0.36 & 0.01 & 0.36 & 0 & 0 & 0 \\
\hline udp & private & SF & 28 & 0 & 3 & 93 & 93 & 0 & 0 & 1 & 0 & 165 & 93 & 0.56 & 0.02 & 0.56 & 0 & 0 & 0 \\
\hline udp & private & SF & 28 & 0 & 3 & 94 & 94 & 0 & 0 & 1 & 0 & 166 & 94 & 0.57 & 0.02 & 0.57 & 0 & 0 & 0 \\
\hline udp & private & SF & 28 & 0 & 3 & 95 & 95 & 0 & 0 & 1 & 0 & 167 & 95 & 0.57 & 0.02 & 0.57 & 0 & 0 & 0 \\
\hline udp & private & SF & 28 & 0 & 3 & 96 & 96 & 0 & 0 & 1 & 0 & 168 & 96 & 0.57 & 0.02 & 0.57 & 0 & 0 & 0 \\
\hline udp & private & SF & 28 & 0 & 3 & 97 & 97 & 0 & 0 & 1 & 0 & 169 & 97 & 0.57 & 0.02 & 0.57 & 0 & 0 & 0 \\
\hline udp & private & SF & 28 & 0 & 3 & 98 & 98 & 0 & 0 & 1 & 0 & 170 & 98 & 0.58 & 0.02 & 0.58 & 0 & 0 & 0 \\
\hline udp & private & SF & 28 & 0 & 3 & 99 & 99 & 0 & 0 & 1 & 0 & 171 & 99 & 0.58 & 0.02 & 0.58 & 0 & 0 & 0 \\
\hline tcp & finger & So & 0 & 1 & 0 & 1 & 1 & 1 & 1 & 1 & 0 & 1 & 8 & 1 & 0 & 1 & 0.38 & 1 & 0.12 \\
\hline
\end{tabular}




\section{System Implementation}

This chapter presents implementation of Association rule classifier model, documentation of the designed system and the user interfaces. The software and hardware requirement needed for the system and also the testing of the system for verification and validation of functions, as well as the result [3-10].

\section{Interface Design}

Start Page: This page is the first page that is seen when the application is executed. The option button enables the use of already generated rules to be reused for classification of another file. If the check box is unselected, the option of selecting a folder containing already generated rule is enabled. Furthermore, select the source file and then the new file to classify. The page is as shown below (Figure 1).

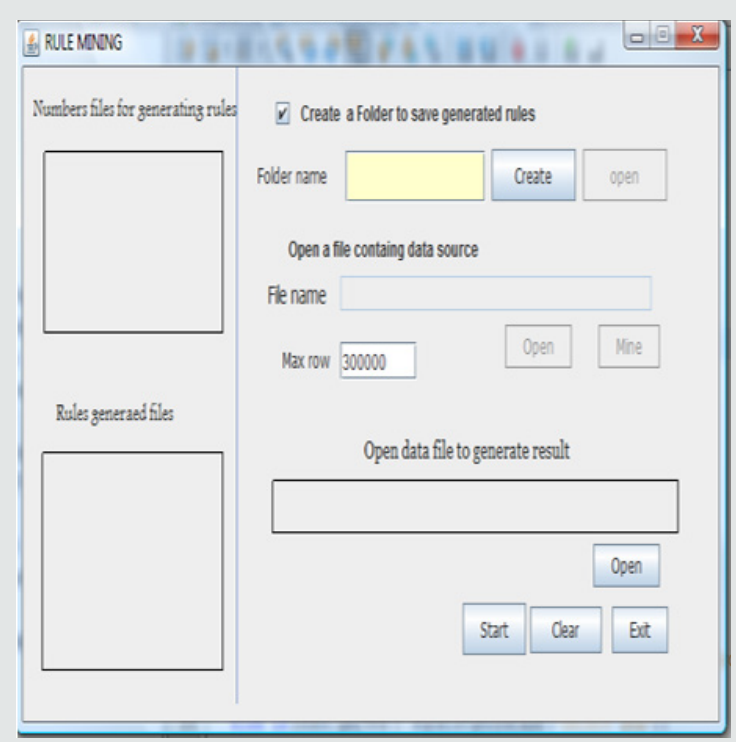

Figure 1: Start page.

Creating Folder: The first step is to create a folder where the rules will be saved, and also the numbers for generating the rules will be saved. This operation is allowed only if the check box on the form is checked, if there is an error with the folder creation process, an error message will be displayed. The open button for the file name is enabled if the folder is created successfully. The interface for the creation of folder is shown below (Figure 2).

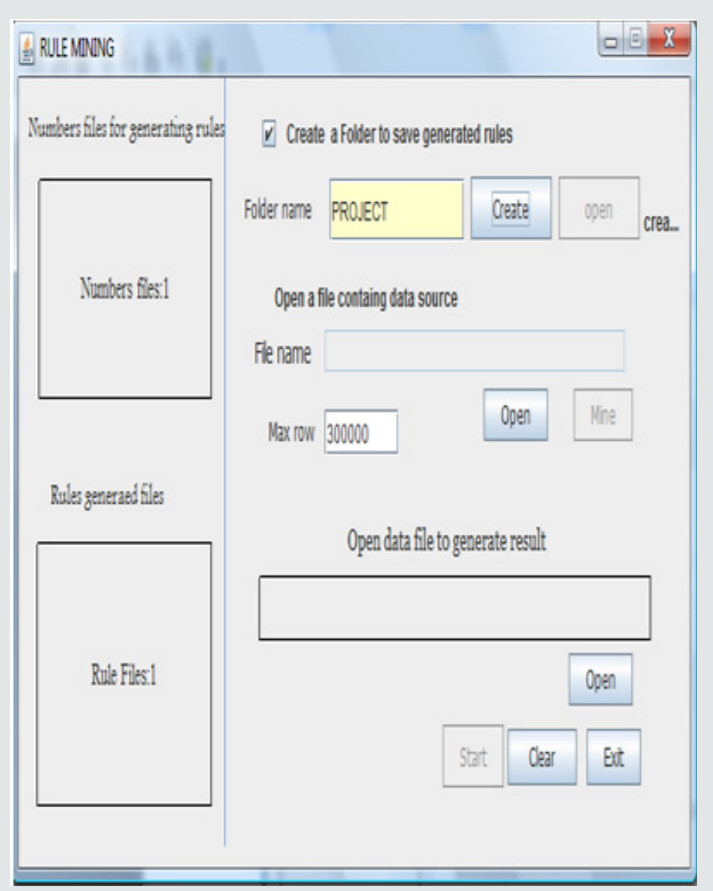

Figure 2: Setting folder name. 
Source File: The source of data for generating the rule is the next requirement. The open button closes to the Mine button enable you to specify the file containing the data from which the rules will be generated. Before the rules are generated from the file, the size of the file is calculated to obtain the number of combination(arrangement) required to generate the rule. The selected source file is seen below (Figure 3).

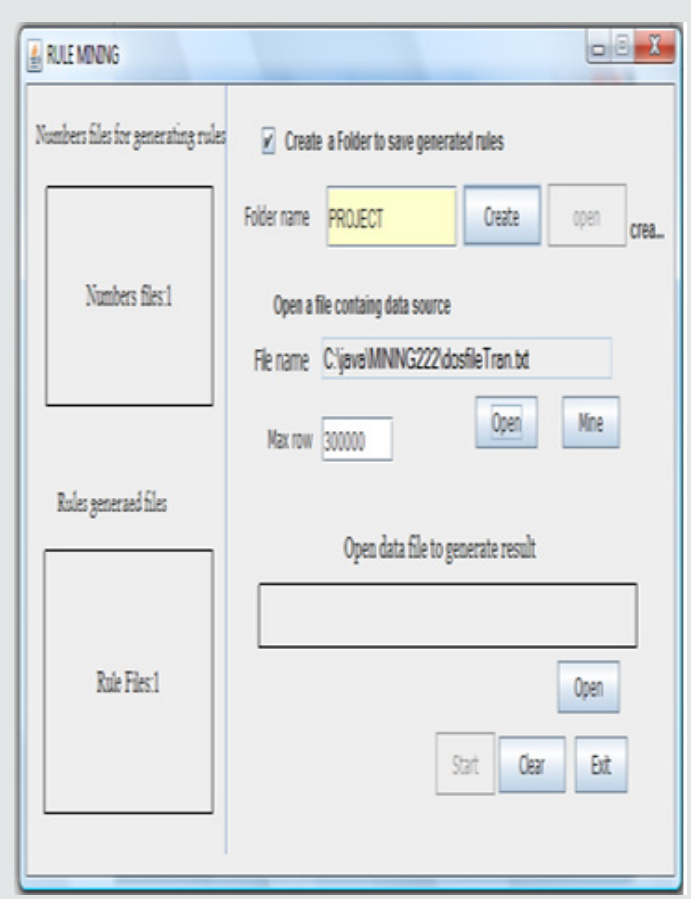

Figure 3: Locating source file.

a) Mining File: The selected source file is mined to extract the rules needed for classification. Depending on the size of the file, it could take a while to complete. On completion, a message dialog is displayed, as shown below (Figure 4).

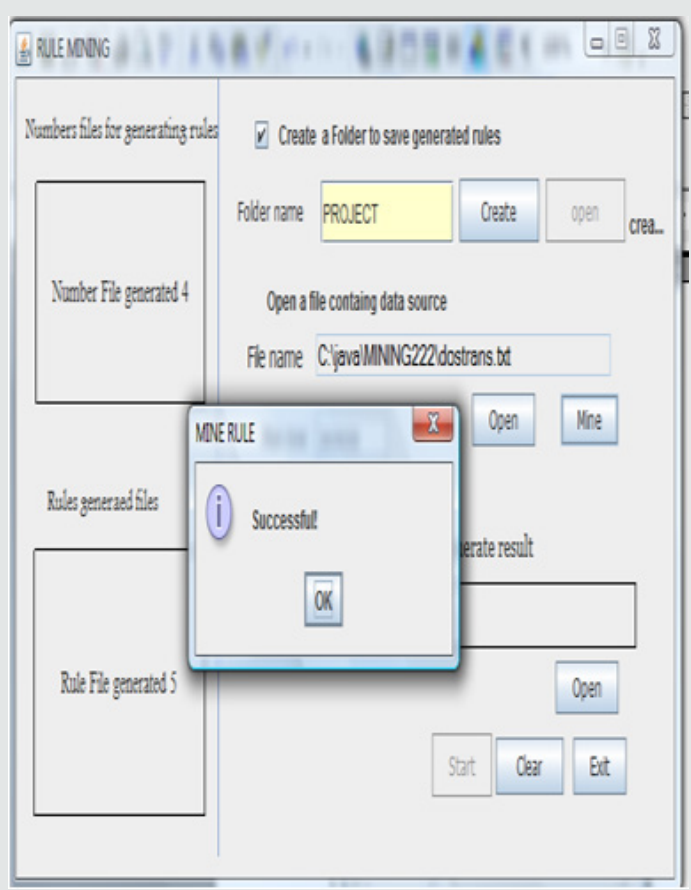

Figure 4: Mining source file.

b) File Classification: After obtaining all the rules from the source file, the open button for selecting a data file to classify is enabled. A file can be classified based on the rules generated. The selected file can be obtained using the open button, as shown below (Figure 5). 


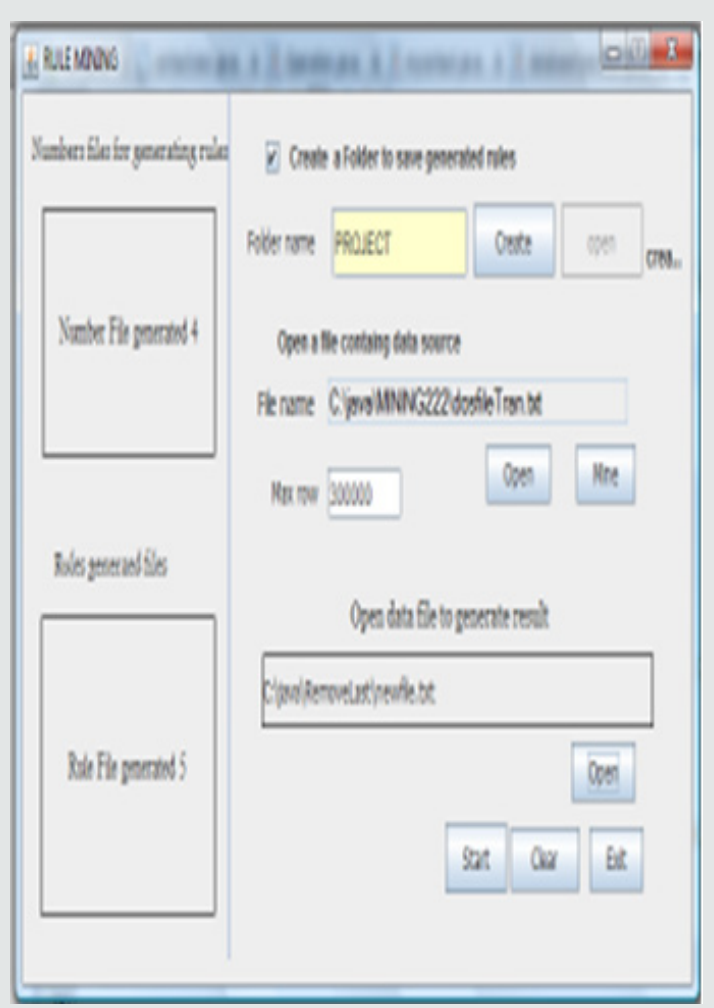

Figure 5: Locating file to classify.

c) Generate Result: Click on the start button to begin the generation of output or result from the selected file to classify, based on the rules generated. The output or result is saved in the folder called result within the folder specified above. Depending on the size of the file to classify, the output might take a while. On completion of the classification, a dialog appears to signify the completion of the classification. This is shown in Figure 6.

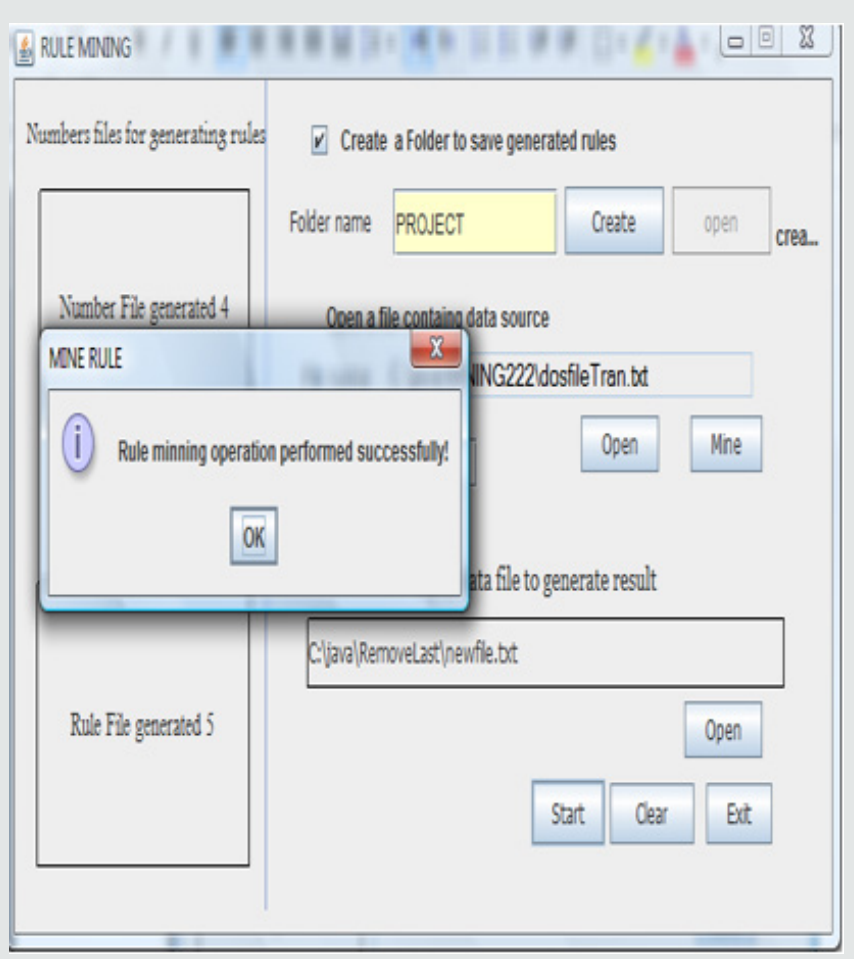

Figure 6: Generating result file from selected file based on rules obtained.

d) Exit Program: Click the Exit button to exit or terminate the application (Figure 7). 


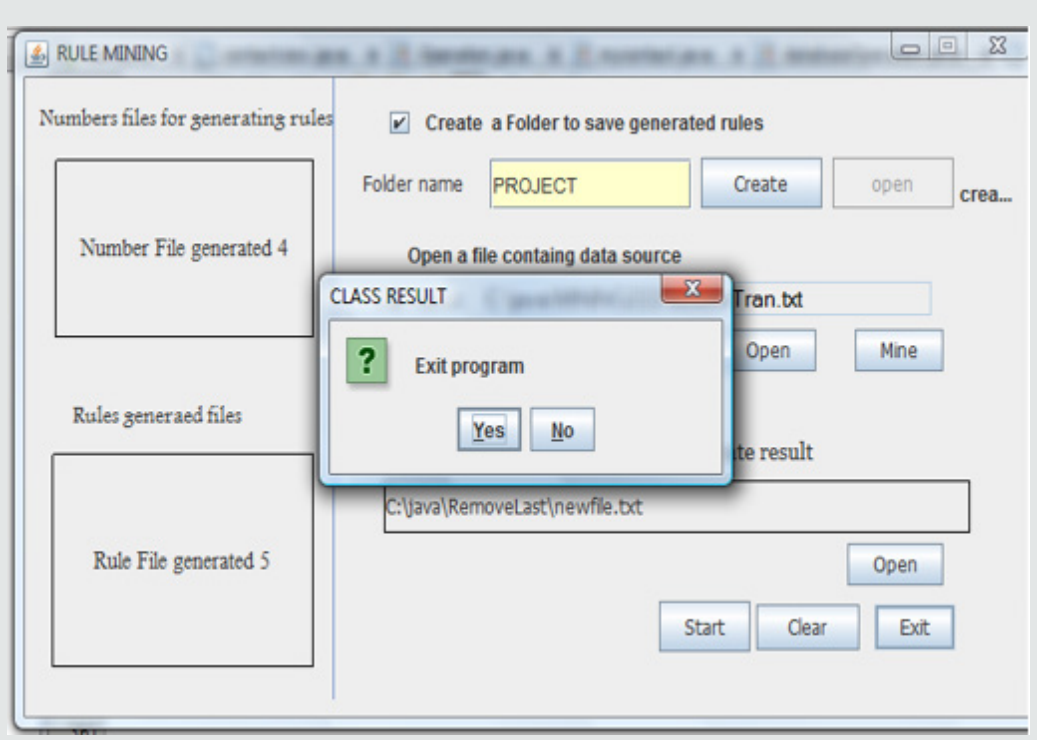

Figure 7: Exit program.

\section{Experimental Setup and Results}

The training dataset consisted of 37,079 records, among which there are $99(.266 \%)$ teardrop, 36,944 (99.66\%) smurf, $20(0.05 \%)$ pod, $15(0.04 \%)$ neptume and $1(0.026 \%)$ land connections. The training dataset use for testing is made up of 400 records out of which there are 98(24.5\%) teardrop, 266(66.5\%) smurf, 2 (5\%) pod, $15(3.75 \%)$ neptume and $1(0.025 \%)$ land while the test dataset is made up of 300 records out of there are $40(13.3 \%)$ Pod, 107 (35.6\%) smurf, 9(3\%) teardrop, 43(14.3\%) neptune (14.3\%), 9(3\%) land, 33(11\%) apache2, 21(7\%) normal, 25(8.3\%) mailbomb and 8(2.6\%) snmpgetattack [11-20].

The test and training data are not from the same probability distribution. In each connection are 20 attributes out of 41 attributes describing different features of the connection (excluding the label attribute)

\section{Results}

Tables 5-14 shows the confusion matrix obtained Association rule mining with 20 attributes.

Table 5: Confusion matrix obtained from one attribute combination from test dataset (unprune rules).

\begin{tabular}{|c|c|c|c|c|c|c|c|c|c|c|}
\hline & \multicolumn{6}{|c|}{ Known Attacks } & \multicolumn{4}{|c|}{ Unknown Attacks } \\
\hline & Pod & Smurf & Teardrop & Neptune & Land & Normal & Appache & Mail bomb & Snmpget & Unknown \\
\hline Pod (40) & 0 & $40(100 \%)$ & 0 & 0 & 0 & 0 & 0 & 0 & 0 & 0 \\
\hline Smurf (107) & 0 & $107(100 \%)$ & 0 & 0 & 0 & 0 & 0 & 0 & 0 & 0 \\
\hline Teardrop (9) & 0 & $9(100 \%)$ & 0 & 0 & 0 & 0 & 0 & 0 & 0 & 0 \\
\hline Neptume (44) & 0 & 0 & 0 & 0 & $44(100 \%)$ & 0 & 0 & 0 & 0 & 0 \\
\hline Land (9) & 0 & 0 & 0 & 0 & $9(100 \%)$ & 0 & 0 & 0 & 0 & 0 \\
\hline Normal (22) & 0 & $3(13.6 \%)$ & 0 & $3(13.6 \%)$ & 0 & $16(72 \%)$ & 0 & 0 & 0 & 0 \\
\hline Appache (34) & 0 & 0 & 0 & $2(6 \%)$ & $32(94 \%)$ & 0 & & 0 & 0 & 0 \\
\hline Mailbomb (26) & 0 & $26(100 \%)$ & 0 & 0 & 0 & 0 & 0 & 0 & 0 & 0 \\
\hline Snmpget(8) & 0 & $8(100 \%)$ & 0 & & 0 & 0 & 0 & 0 & 0 & 0 \\
\hline
\end{tabular}


Table 6: Confusion matrix obtained from one attribute combination from test dataset (prune rules).

\begin{tabular}{|c|c|c|c|c|c|c|c|c|c|c|}
\hline & Pod & Smurf & Teardrop & Neptune & Land & Normal & Appache & Mail bomb & Snmpget & Unknown \\
\hline Pod (40) & $4(10 \%)$ & 0 & $34(85 \%)$ & 0 & 0 & 0 & 0 & 0 & 0 & $2(5 \%)$ \\
\hline Smurf (107) & 0 & $103(96.3 \%)$ & 0 & 0 & 0 & 0 & 0 & 0 & 0 & $4(3.7 \%)$ \\
\hline Teardrop (9) & 0 & $9(100 \%)$ & 0 & 0 & 0 & 0 & 0 & 0 & 0 & 0 \\
\hline Neptume (44) & $1(2.2 \%)$ & 0 & $3(6.8 \%)$ & $9(20.5 \%)$ & $1(2.2 \%) 5$ & 0 & 0 & $1(2.2 \%)$ & 0 & $29(65.9 \%)$ \\
\hline Land (9) & 0 & 0 & $1(11.1 \%)$ & $1(11.1 \%)$ & $7(77.7 \%)$ & 0 & 0 & 0 & 0 & 0 \\
\hline Normal (22) & 0 & 0 & 0 & $2(9 \%)$ & 0 & $20(91 \%)$ & 0 & 0 & 0 & 0 \\
\hline Appache (34) & 0 & 0 & $1(2.9 \%)$ & 0 & $3(8.82 \%)$ & 0 & & 0 & 0 & $29(85.2 \%)$ \\
\hline $\begin{array}{c}\text { Mailbomb } \\
\text { (26) }\end{array}$ & 0 & 0 & $14(53.8 \%)$ & 0 & 0 & 0 & 0 & 0 & 0 & $12(46.1 \%)$ \\
\hline Snmpget(8) & 0 & $8(100 \%)$ & 0 & & 0 & 0 & 0 & 0 & 0 & 0 \\
\hline
\end{tabular}

Table 7: Confusion matrix obtained from one and two attribute combination from test dataset (unprune rules).

\begin{tabular}{|c|c|c|c|c|c|c|c|c|c|c|}
\hline & Pod & Smurf & Teardrop & Neptune & Land & Normal & Appache & Mail bomb & Snmpget & Unknown \\
\hline Pod (40) & 0 & 0 & 0 & 0 & $40(10 \%)$ & 0 & 0 & 0 & 0 & 0 \\
\hline Smurf (107) & 0 & $107(100 \%)$ & 0 & 0 & 0 & 0 & 0 & 0 & 0 & 0 \\
\hline Teardrop (9) & 0 & 0 & $7(88 \%)$ & 0 & $2(12 \%)$ & 0 & 0 & 0 & 0 & 0 \\
\hline Neptume (44) & 0 & 0 & 0 & 0 & $44(10 \%)$ & 0 & 0 & 0 & 0 & 0 \\
\hline Land (9) & 0 & 0 & 0 & 0 & $9(100 \%)$ & 0 & 0 & 0 & 0 & 0 \\
\hline Normal (22) & 0 & $3(13.6 \%)$ & 0 & $3(13.6 \%)$ & 0 & $16(72 \%)$ & 0 & 0 & 0 & 0 \\
\hline Appache (34) & 0 & 0 & 0 & 0 & $34(10 \%)$ & 0 & 0 & 0 & 0 & 0 \\
\hline $\begin{array}{c}\text { Mailbomb } \\
\text { (26) }\end{array}$ & 0 & 0 & 0 & 0 & $26(10 \%)$ & 0 & 0 & 0 & 0 & 0 \\
\hline Snmpget(8) & 0 & $8(100 \%)$ & 0 & & 0 & 0 & 0 & 0 & 0 & 0 \\
\hline
\end{tabular}

Table 8: Confusion matrix obtained from one and two attribute combination from test dataset (prune rules).

\begin{tabular}{|c|c|c|c|c|c|c|c|c|c|c|}
\hline & Pod & Smurf & Teardrop & Neptune & Land & Normal & Appache & Mail bomb & Snmpget & Unknown \\
\hline Pod (40) & 0 & $40(100 \%)$ & 0 & 0 & 0 & 0 & 0 & 0 & 0 & 0 \\
\hline Smurf (107) & 0 & $107(100 \%)$ & 0 & 0 & 0 & 0 & 0 & 0 & 0 & 0 \\
\hline Teardrop (9) & 0 & $2(12 \%)$ & $7(88 \%)$ & 0 & 0 & 0 & 0 & 0 & 0 & 0 \\
\hline Neptume (44) & 0 & 0 & 0 & $44(100 \%)$ & 0 & 0 & 0 & 0 & 0 & 0 \\
\hline Land (9) & 0 & 0 & 0 & 0 & $9(100 \%)$ & 0 & 0 & 0 & 0 & 0 \\
\hline Normal (22) & 0 & 0 & 0 & $2(9 \%)$ & 0 & $20(91 \%)$ & 0 & 0 & 0 & 0 \\
\hline Appache (34) & 0 & $1(2.9 \%)$ & 0 & $1(2.9 \%)$ & 0 & 0 & 0 & 0 & 0 & $32(94.1 \%)$ \\
\hline
\end{tabular}

Table 9: Confusion matrix obtained from one, two and three attribute combination from test dataset (unprune rules).

\begin{tabular}{|c|c|c|c|c|c|c|c|c|c|c|}
\hline & Pod & Smurf & Teardrop & Neptune & Land & Normal & Appache & Mail bomb & Snmpget & Unknown \\
\hline Pod (40) & 0 & $40(100 \%)$ & 0 & 0 & 0 & 0 & 0 & 0 & 0 & 0 \\
\hline Smurf (107) & 0 & $107(100 \%)$ & 0 & 0 & 0 & 0 & 0 & 0 & 0 & 0 \\
\hline Teardrop (9) & 0 & 0 & $9(100 \%)$ & 0 & 0 & 0 & 0 & 0 & 0 & 0 \\
\hline Neptume (44) & 0 & 0 & 0 & 0 & $44(100 \%)$ & 0 & 0 & 0 & 0 & 0 \\
\hline Land (9) & 0 & 0 & 0 & 0 & $9(100 \%)$ & 0 & 0 & 0 & 0 & 0 \\
\hline Normal (22) & 0 & $2(9 \%)$ & 0 & $3(13.6 \%)$ & 0 & $17(77 \%)$ & 0 & 0 & 0 & 0 \\
\hline Appache (34) & 0 & 0 & 0 & 0 & $34(10 \%)$ & 0 & 0 & 0 & 0 & 0 \\
\hline Mailbomb (26) & 0 & 0 & 0 & 0 & $26(10 \%)$ & 0 & 0 & 0 & 0 & 0 \\
\hline Snmpget(8) & 0 & $8(100 \%)$ & 0 & & 0 & 0 & 0 & 0 & 0 & 0 \\
\hline
\end{tabular}


Table 10: Confusion matrix obtained from one, two and three attribute combination from test dataset (prune rules).

\begin{tabular}{|c|c|c|c|c|c|c|c|c|c|c|}
\hline & Pod & Smurf & Teardrop & Neptune & Land & Normal & Appache & Mail bomb & Snmpget & Unknown \\
\hline Pod (40) & $38(95 \%)$ & $2(5 \%)$ & 0 & 0 & 0 & 0 & 0 & 0 & 0 & 0 \\
\hline Smurf (107) & 0 & $107(100 \%)$ & 0 & 0 & 0 & 0 & 0 & 0 & 0 & 0 \\
\hline Teardrop (9) & 0 & 0 & $9(100 \%)$ & 0 & 0 & 0 & 0 & 0 & 0 & 0 \\
\hline Neptume (44) & 0 & 0 & 0 & $44(100 \%)$ & 0 & 0 & 0 & 0 & 0 & 0 \\
\hline Land (9) & 0 & 0 & 0 & 0 & $9(100 \%)$ & 0 & 0 & 0 & 0 & 0 \\
\hline Normal (22) & 0 & 0 & 0 & $2(9 \%)$ & 0 & $20(91 \%)$ & 0 & 0 & 0 & 0 \\
\hline Appache (34) & 0 & $1(2.9 \%)$ & 0 & $1(2.9 \%)$ & 0 & 0 & 0 & 0 & 0 & $32(94.1 \%)$ \\
\hline Mailbomb (26) & 0 & $10(138.5 \%)$ & 0 & 0 & 0 & 0 & 0 & 0 & 0 & $16(61.5 \%)$ \\
\hline Snmpget(8) & 0 & $3(37.5 \%)$ & 0 & & 0 & 0 & 0 & 0 & 0 & $5(62.5 \%)$ \\
\hline
\end{tabular}

Table 11: Confusion matrix obtained from one, two, three and four attribute combination from test dataset (unprune rules).

\begin{tabular}{|c|c|c|c|c|c|c|c|c|c|c|}
\hline & Pod & Smurf & Teardrop & Neptune & Land & Normal & Appache & Mail bomb & Snmpget & Unknown \\
\hline Pod (40) & 0 & $40(10 \%)$ & 0 & 0 & 0 & 0 & 0 & 0 & 0 & 0 \\
\hline Smurf (107) & 0 & $107(100 \%)$ & 0 & 0 & 0 & 0 & 0 & 0 & 0 & 0 \\
\hline Teardrop (9) & 0 & 0 & $9(100 \%)$ & 0 & 0 & 0 & 0 & 0 & 0 & 0 \\
\hline Neptume (44) & 0 & 0 & 0 & $10(22.7 \%)$ & $34(77.8 \%)$ & 0 & 0 & 0 & 0 & 0 \\
\hline Land (9) & 0 & 0 & 0 & 0 & $9(100 \%)$ & 0 & 0 & 0 & 0 & 0 \\
\hline Normal (22) & 0 & $2(9)$ & 0 & $3(13.6 \%)$ & 0 & $17(77 \%)$ & 0 & 0 & 0 & 0 \\
\hline Appache (34) & 0 & 0 & 0 & 0 & $34(100 \%)$ & 0 & 0 & 0 & 0 & 0 \\
\hline $\begin{array}{c}\text { Mailbomb } \\
(26)\end{array}$ & 0 & 0 & 0 & 0 & $26(100 \%)$ & 0 & 0 & 0 & 0 & 0 \\
\hline Snmpget(8) & 0 & 0 & $8(100 \%)$ & & 0 & 0 & 0 & 0 & 0 & 0 \\
\hline
\end{tabular}

Table 12: Confusion matrix obtained from one, two, three and four attribute combination from test dataset (prune rules).

\begin{tabular}{|c|c|c|c|c|c|c|c|c|c|c|}
\hline & Pod & Smurf & Teardrop & Neptune & Land & Normal & Appache & Mail bomb & Snmpget & Unknown \\
\hline Pod (40) & $38(95 \%)$ & $2(5 \%)$ & 0 & 0 & 0 & 0 & 0 & 0 & 0 & 0 \\
\hline Smurf (107) & 0 & $107(100 \%)$ & 0 & 0 & 0 & 0 & 0 & 0 & 0 & 0 \\
\hline Teardrop (9) & 0 & 0 & $9(100 \%)$ & 0 & 0 & 0 & 0 & 0 & 0 & 0 \\
\hline Neptume (44) & 0 & 0 & 0 & $44(100 \%)$ & 0 & 0 & 0 & 0 & 0 & 0 \\
\hline Land (9) & 0 & 0 & 0 & 0 & $9(100 \%)$ & 0 & 0 & 0 & 0 & 0 \\
\hline Normal (22) & 0 & 0 & 0 & $2(9 \%)$ & 0 & $20(91 \%)$ & 0 & 0 & 0 & 0 \\
\hline Appache (34) & 0 & $1(2.9 \%)$ & 0 & $1(2.9 \%)$ & 0 & 0 & 0 & 0 & 0 & $32(94.1 \%)$ \\
\hline Mailbomb (26) & 0 & $10(138.5 \%)$ & 0 & 0 & 0 & 0 & 0 & 0 & 0 & $16(61.5 \%)$ \\
\hline Snmpget(8) & 0 & $3(37.5 \%)$ & 0 & & 0 & 0 & 0 & 0 & 0 & $5(62.5 \%)$ \\
\hline
\end{tabular}

Table 13: Confusion matrix obtained from one, two, three, four and five attribute combination from test dataset (unprune rules).

\begin{tabular}{|c|c|c|c|c|c|c|c|c|c|c|}
\hline & Pod & Smurf & Teardrop & Neptune & Land & Normal & Appache & Mail bomb & Snmpget & Unknown \\
\hline Pod (40) & $40(100 \%)$ & 0 & 0 & 0 & 0 & 0 & 0 & 0 & 0 & 0 \\
\hline Smurf (107) & 0 & $107(100 \%)$ & 0 & 0 & 0 & 0 & 0 & 0 & 0 & 0 \\
\hline Teardrop (9) & 0 & 0 & $9(100 \%)$ & 0 & 0 & 0 & 0 & 0 & 0 & 0 \\
\hline Neptume (44) & 0 & 0 & 0 & $10(22.7 \%)$ & $34(77.8 \%)$ & 0 & 0 & 0 & 0 & 0 \\
\hline Land (9) & 0 & 0 & 0 & 0 & $9(100 \%)$ & 0 & 0 & 0 & 0 & 0 \\
\hline Normal (22) & 0 & $2(9 \%)$ & 0 & $3(13.6 \%)$ & 0 & $17(77 \%)$ & 0 & 0 & 0 & 0 \\
\hline Appache (34) & 0 & 0 & 0 & 0 & $34(100 \%)$ & 0 & 0 & 0 & 0 & 0 \\
\hline Mailbomb (26) & 0 & 0 & 0 & 0 & $26(100 \%)$ & 0 & 0 & 0 & 0 & 0 \\
\hline Snmpget(8) & 0 & $8(100 \%)$ & 0 & & 0 & 0 & 0 & 0 & 0 & 0 \\
\hline
\end{tabular}


Table 14: Confusion matrix obtained from one, two, three, four and five attribute combination from test dataset (prune rules).

\begin{tabular}{|c|c|c|c|c|c|c|c|c|c|c|}
\hline & Pod & Smurf & Teardrop & Neptune & Land & Normal & Appache & Mail bomb & Snmpget & Unknown \\
\hline Pod (40) & $38(95 \%)$ & $2(5 \%)$ & 0 & 0 & 0 & 0 & 0 & 0 & 0 & 0 \\
\hline Smurf (107) & 0 & $107(100 \%)$ & 0 & 0 & 0 & 0 & 0 & 0 & 0 & 0 \\
\hline Teardrop (9) & 0 & 0 & $9(100 \%)$ & 0 & 0 & 0 & 0 & 0 & 0 & 0 \\
\hline Neptume (44) & 0 & 0 & 0 & $14(100 \%)$ & 0 & 0 & 0 & 0 & 0 & 0 \\
\hline Land (9) & 0 & 0 & 0 & 0 & $9(100 \%)$ & 0 & 0 & 0 & 0 & 0 \\
\hline Normal (22) & 0 & 0 & 0 & $2(9 \%)$ & 0 & $20(91 \%)$ & 0 & 0 & 0 & 0 \\
\hline Appache (34) & 0 & $1(2.9 \%)$ & 0 & $1(2.9 \%)$ & 0 & 0 & 0 & 0 & 0 & $32(94.1 \%)$ \\
\hline Mailbomb (26) & 0 & $10(138.5 \%)$ & 0 & 0 & 0 & 0 & 0 & 0 & 0 & $16(61.5 \%)$ \\
\hline Snmpget(8) & 0 & $3(37.5 \%)$ & 0 & & 0 & 0 & 0 & 0 & 0 & $5(62.5 \%)$ \\
\hline
\end{tabular}

\section{Discussion}

The results in Tables 15-19, were obtained from classification of training data set with raw unprune rule set, from the tables, the degree of accuracy of classification of smurf attack ranges between $99.6 \%$ to $100 \%$. Pod attacks classification could not be classified correct by the classification model, about $95 \%$ pod attacks were classified as smurf attacks, while the rest were classified as Pod.
$98 \%$ of teardrop and $100 \%$ of land attacks were also correctly classify, while less than $20 \%$ of Neptune attacks were classified correctly using rules based on 0 ne, two and three combinational attributes, rules based on four and five combinational attributes has better performance of classification of Neptune attacks (65\%) than one - three attributes combination. Table 20 shows the summary of all the attacks correctly classified in Tables 15-19.

Table 15: Confusion matrix obtained from one attribute combination from training dataset (unprune rules).

\begin{tabular}{|c|c|c|c|c|c|}
\hline Predicted as Actual & Neptune & Smurf & Pod & Teardrop & Land \\
\hline Neptune (16) & $14(87.5 \%)$ & $0.00 \%$ & $0.00 \%$ & $0.00 \%$ & $2(12.5 \%)$ \\
\hline Smurf (264) & $0(0.00) \%$ & $264(100 \%)$ & $0(0.00 \%)$ & $0(0.00 \%)$ & $0(0.00 \%)$ \\
\hline Pod (20) & $0(0.00) \%$ & $20(100 \%)$ & $0(0.00) \%$ & $0(0.00) \%$ & $0(0.00) \%$ \\
\hline Teardrop (99) & $0(0.00) \%$ & $99(100 \%)$ & $0(0.00) \%$ & $0(0.00) \%$ & $0(0.00) \%$ \\
\hline Land (1) & $0(0.00) \%$ & $0(0.00) \%$ & $0(0.00) \%$ & $0(0.00) \%$ & $1(100 \%)$ \\
\hline
\end{tabular}

Table 16: Confusion matrix obtained from one and two attribute combination from training dataset (unprune rules).

\begin{tabular}{|c|c|c|c|c|c|}
\hline Predicted as Actual & Neptune & Smurf & Pod & Teardrop & Land \\
\hline Neptune (16) & $\mathbf{1 2}(\mathbf{7 5 .} \%)$ & $0.00 \%$ & $0.00 \%$ & $0(0.00 \%)$ & $4(25 \%)$ \\
\hline Smurf (264) & $0(0.00) \%$ & $\mathbf{2 6 3}(\mathbf{9 9 . 6 2 \% )}$ & $0(0.00 \%)$ & $1(0.37 \%)$ & $0(0.00 \%)$ \\
\hline Pod (20) & $0(0.00) \%$ & $19(95 \%)$ & $\mathbf{( 0 . 0 )} \%$ & $0(0.00) \%$ & $1(5.00) \%$ \\
\hline Teardrop $(99)$ & $0(0.00) \%$ & $99(100 \%)$ & $0(0.00) \%$ & $\mathbf{0 ( 0 . 0 0 )} \%$ & $0(0.00) \%$ \\
\hline Land $(1)$ & $0(0.00) \%$ & $0(0.00) \%$ & $0(0.00) \%$ & $0(0.00) \%$ & $\mathbf{1 ( 1 0 0 \% )}$ \\
\hline
\end{tabular}

Table 17: Confusion matrix obtained from one, two and three attribute combination from training dataset (unprune rules).

\begin{tabular}{|c|c|c|c|c|c|}
\hline Predicted as Actual & Neptune & Smurf & Pod & Teardrop & Land \\
\hline Neptune (16) & $0(0.00 \%)$ & $0.00 \%$ & $0.00 \%$ & $0(0.00 \%)$ & $16(100 \%)$ \\
\hline Smurf (264) & $0(0.00) \%$ & $264(100 \%)$ & $0(0.00 \%)$ & $0(0.00 \%)$ & $0(0.00 \%)$ \\
\hline Pod (20) & $0(0.00) \%$ & $19(95 \%)$ & $1(5.00) \%$ & $0(0.00) \%$ & $0(0.00) \%$ \\
\hline Teardrop (99) & $0(0.00) \%$ & $0(0.00 \%)$ & $0(0.00) \%$ & $99(100) \%$ & $0(0.00) \%$ \\
\hline Land (1) & $0(0.00) \%$ & $0(0.00) \%$ & $0(0.00) \%$ & $0(0.00) \%$ & $1(100 \%)$ \\
\hline
\end{tabular}


Table 18: Confusion matrix obtained from one, two, three and four attributes combination from training dataset (unprune rules).

\begin{tabular}{|c|c|c|c|c|c|}
\hline Predicted as Actual & Neptune & Smurf & Pod & Teardrop & Land \\
\hline Neptune (16) & $14(87.5 \%)$ & $0.00 \%$ & $0.00 \%$ & $0(0.00 \%)$ & $2(12.5 \%)$ \\
\hline Smurf (264) & $0(0.00) \%$ & $264(100 \%)$ & $0(0.00 \%)$ & $0(0.00 \%)$ & $0(0.00 \%)$ \\
\hline Pod (20) & $0(0.00) \%$ & $19(95 \%)$ & $1(5.00) \%$ & $0(0.00) \%$ & $0(0.00) \%$ \\
\hline Teardrop (99) & $0(0.00) \%$ & $0(0.00 \%)$ & $0(0.00) \%$ & $99(100) \%$ & $0(0.00) \%$ \\
\hline Land $(1)$ & $0(0.00) \%$ & $0(0.00) \%$ & $0(0.00) \%$ & $0(0.00) \%$ & $1(100 \%)$ \\
\hline
\end{tabular}

Table 19: Confusion matrix obtained from one, two, three, four and five attributes combination from training dataset (unprune rules).

\begin{tabular}{|c|c|c|c|c|c|}
\hline Predicted as Actual & Neptune & Smurf & Pod & Teardrop & Land \\
\hline Neptune (16) & $14(87.5 \%)$ & $0.00 \%$ & $0.00 \%$ & $0(0.00 \%)$ & $2(12.5 \%)$ \\
\hline Smurf (264) & $0(0.00) \%$ & $264(100 \%)$ & $0(0.00 \%)$ & $0(0.00 \%)$ & $0(0.00 \%)$ \\
\hline Pod (20) & $0(0.00) \%$ & $19(95 \%)$ & $1(5.00) \%$ & $0(0.00) \%$ & $0(0.00) \%$ \\
\hline Teardrop (99) & $0(0.00) \%$ & $0(0.00 \%)$ & $0(0.00) \%$ & $99(100) \%$ & $0(0.00) \%$ \\
\hline Land $(1)$ & $0(0.00) \%$ & $0(0.00) \%$ & $0(0.00) \%$ & $0(0.00) \%$ & $1(100 \%)$ \\
\hline
\end{tabular}

Table 20: Percentages of Correctly Classified Attacks in Table 15-19.

\begin{tabular}{|c|c|c|c|c|c|}
\hline & Neptune & Smurf & Pod & Teardrop & Land \\
\hline Table 15 & $87.5 \%$ & $100 \%$ & $0 \%$ & $0 \%$ & $1000 \%$ \\
\hline Table 16 & $75 \%$ & $99.6 \%$ & $0 \%$ & $0 \%$ & $100 \%$ \\
\hline Table 17 & $87.5 \% .5 \%$ & $100 \%$ & $5 \%$ & $99 \%$ & $100 \%$ \\
\hline Table 18 & $87.5 \%$ & $100 \%$ & $5 \%$ & $100 \%$ & $100 \%$ \\
\hline Table 19 & $87.5 \%$ & $100 \%$ & $5 \%$ & $100 \%$ & $100 \%$ \\
\hline
\end{tabular}

Table 21: Confusion matrix obtained from one attribute combination from training dataset (prune rules).

\begin{tabular}{|c|c|c|c|c|c|}
\hline Predicted as Actual & Neptune & Smurf & Pod & Teardrop & Land \\
\hline Neptune (16) & $\mathbf{1 1 ( 6 8 . 7 5 \% )}$ & $0.00 \%$ & $0.00 \%$ & $1(6.25 \%$ & $3(18.75 \%)$ \\
\hline Smurf (264) & $0(0.00) \%$ & $\mathbf{2 6 3 ( 9 9 . 6 2 \% )}$ & $0(0.00 \%)$ & $1(0.37 \%)$ & $0(0.00 \%)$ \\
\hline Pod (20) & $0(0.00) \%$ & $0(0.00 \%)$ & $\mathbf{1 8 ( 9 0 )} \%$ & $1(5.00) \%$ & $1(5.00) \%$ \\
\hline Teardrop $(99)$ & $0(0.00) \%$ & $0(0.00 \%)$ & $0(0.00) \%$ & $\mathbf{9 9}(\mathbf{1 0 0}) \%$ & $0(0.00) \%$ \\
\hline Land $(1)$ & $0(0.00) \%$ & $0(0.00) \%$ & $0(0.00) \%$ & $0(0.00) \%$ & $\mathbf{1 ( 1 0 0 \% )}$ \\
\hline
\end{tabular}

Table 22: Confusion matrix obtained from one and attribute combination from training dataset (prune rules).

\begin{tabular}{|c|c|c|c|c|c|}
\hline Predicted as Actual & Neptune & Smurf & Pod & Teardrop & Land \\
\hline Neptune (16) & $\mathbf{1 4}(\mathbf{9 3 . 7 5 . \% )}$ & $0.00 \%$ & $0.00 \%$ & $0(0.00 \%)$ & $1(6.25 \%)$ \\
\hline Smurf (264) & $0(0.00) \%$ & $\mathbf{2 6 4}(\mathbf{1 0 0 \% )}$ & $0(0.00 \%)$ & $0(0.00 \%)$ & $0(0.00 \%)$ \\
\hline Pod (20) & $0(0.00) \%$ & $0(100 \%)$ & $\mathbf{2 0 ( 1 0 0 )} \%$ & $0(0.00) \%$ & $0(0.00) \%$ \\
\hline Teardrop (99) & $0(0.00) \%$ & $0(0 \%)$ & $0(0.00) \%$ & $\mathbf{0 ( 1 0 0 )} \%$ & $0(0.00) \%$ \\
\hline Land $(1)$ & $0(0.00) \%$ & $0(0.00) \%$ & $0(0.00) \%$ & $0(0.00) \%$ & $\mathbf{1 ( 1 0 0 \% )}$ \\
\hline
\end{tabular}

Table 23: Confusion matrix obtained from one, two and three attribute combination from training dataset (prune rules).

\begin{tabular}{|c|c|c|c|c|c|}
\hline Predicted as Actual & Neptune & Smurf & Pod & Teardrop & Land \\
\hline Neptune (16) & $\mathbf{1 5}(\mathbf{9 3 . 7 5 \% )}$ & $0.00 \%$ & $0.00 \%$ & $0(0.00 \%)$ & $1(6.25 \%)$ \\
\hline Smurf (264) & $0(0.00) \%$ & $\mathbf{2 6 4}(\mathbf{1 0 0} \%)$ & $0(0.00 \%)$ & $0(0.00 \%)$ & $0(0.00 \%)$ \\
\hline Pod (20) & $0(0.00) \%$ & $0(0.00 \%)$ & $\mathbf{2 0}(\mathbf{1 0 0} \%)$ & $0(0.00) \%$ & $0(0.00) \%$ \\
\hline Teardrop (99) & $0(0.00) \%$ & $0(0.00 \%)$ & $0(0.00) \%$ & $\mathbf{9 9}(\mathbf{1 0 0}) \%$ & $0(0.00) \%$ \\
\hline Land $(1)$ & $0(0.00) \%$ & $0(0.00) \%$ & $0(0.00) \%$ & $0(0.00) \%$ & $\mathbf{1 ( 1 0 0 \% )}$ \\
\hline
\end{tabular}


Table 24: Confusion matrix obtained from one, two, three and four attributes combination from training dataset (prune rules).

\begin{tabular}{|c|c|c|c|c|c|}
\hline Predicted as Actual & Neptune & Smurf & Pod & Teardrop & Land \\
\hline Neptune (16) & $16(100 . \%)$ & $0.00 \%$ & $0.00 \%$ & $0(0.00 \%)$ & $2(12.5 \%)$ \\
\hline Smurf (264) & $0(0.00) \%$ & $264(100 \%)$ & $0(0.00 \%)$ & $0(0.00 \%)$ & $0(0.00 \%)$ \\
\hline Pod (20) & $0(0.00) \%$ & $19(95 \%)$ & $20(100) \%$ & $0(0.00) \%$ & $0(0.00) \%$ \\
\hline Teardrop (99) & $0(0.00) \%$ & $0(0.00 \%)$ & $0(0.00) \%$ & $99(100) \%$ & $0(0.00) \%$ \\
\hline Land (1) & $0(0.00) \%$ & $0(0.00) \%$ & $0(0.00) \%$ & $0(0.00) \%$ & $1(100 \%)$ \\
\hline
\end{tabular}

Table 25: Confusion matrix obtained from one, two, three, four and five attributes combination from training dataset (prune rules).

\begin{tabular}{|c|c|c|c|c|c|}
\hline Predicted as Actual & Neptune & Smurf & Pod & Teardrop & Land \\
\hline Neptune (16) & $\mathbf{1 6}(\mathbf{1 0 0} \%)$ & $0.00 \%$ & $0.00 \%$ & $0(0.00 \%)$ & $0(0.00 \%)$ \\
\hline Smurf (264) & $0(0.00) \%$ & $\mathbf{2 6 4}(\mathbf{1 0 0} \%)$ & $0(0.00 \%)$ & $0(0.00 \%)$ & $0(0.00 \%)$ \\
\hline Pod (20) & $0(0.00) \%$ & $0(0.00 \%)$ & $\mathbf{2 0}(\mathbf{1 0 0}) \%$ & $0(0.00) \%$ & $0(0.00) \%$ \\
\hline Teardrop (99) & $0(0.00) \%$ & $0(0.00 \%)$ & $0(0.00) \%$ & $\mathbf{9 9}(\mathbf{1 0 0} \%)$ & $0(0.00) \%$ \\
\hline Land $(1)$ & $0(0.00) \%$ & $0(0.00) \%$ & $0(0.00) \%$ & $0(0.00) \%$ & $\mathbf{1 ( 1 0 0 \% )}$ \\
\hline
\end{tabular}

Table 26: Percentages of Correctly Classified Attacks in Table 21-25 (prune rule).

\begin{tabular}{|c|c|c|c|c|c|}
\hline & Neptune & Smurf & Pod & Teardrop & Land \\
\hline Table 21 & $87.5 \%$ & $100 \%$ & $90 \%$ & $100 \%$ & $1000 \%$ \\
\hline Table 22 & $75 \%$ & $99.6 \%$ & $100 \%$ & $100 \%$ & $100 \%$ \\
\hline Table 23 & $87.5 \% .5 \%$ & $100 \%$ & $100 \%$ & $100 \%$ & $100 \%$ \\
\hline Table 24 & $87.5 \%$ & $100 \%$ & $100 \%$ & $100 \%$ & $100 \%$ \\
\hline Table 25 & $87.5 \%$ & $100 \%$ & $100 \%$ & $100 \%$ & $100 \%$ \\
\hline
\end{tabular}

The results in Tables 21-25, were obtained from classification of training data set with pruned rule set, the pruned rule set gives a better results than the unpruned data set. All the attacks types except Neptune and pod were correctly classified (100\%) for all the rules categories, pod was $90 \%$ correctly classifier with single attributes rules and $100 \%$ correctly classified with other four categories of rules. Neptune recorded $100 \%$ correct classification for 4 and 5 attributes combinational rules, 93\% correct classification for 2 and 3 attributes combinational rules and 69\% correctly classified for

\section{Discussion}

Table 27: Confusion matrix obtained from one attribute combination from test dataset (unprune rules).

\begin{tabular}{|c|c|c|c|c|c|c|c|c|c|c|}
\hline & \multicolumn{6}{|c|}{ Known Attacks } & \multicolumn{4}{|c|}{ Unknown Attacks } \\
\hline & Pod & Smurf & Teardrop & Neptune & Land & Normal & Appache & Mail bomb & Snmpget & Unknown \\
\hline Pod (40) & 0 & $40(100 \%)$ & 0 & 0 & 0 & 0 & 0 & 0 & 0 & 0 \\
\hline Smurf (107) & 0 & $107(100 \%)$ & 0 & 0 & 0 & 0 & 0 & 0 & 0 & 0 \\
\hline Teardrop (9) & 0 & $9(100 \%)$ & 0 & 0 & 0 & 0 & 0 & 0 & 0 & 0 \\
\hline $\begin{array}{l}\text { Neptume } \\
(44)\end{array}$ & 0 & 0 & 0 & 0 & $44(100 \%)$ & 0 & 0 & 0 & 0 & 0 \\
\hline Land (9) & 0 & 0 & 0 & 0 & $9(100 \%)$ & 0 & 0 & 0 & 0 & 0 \\
\hline Normal (22) & 0 & $3(13.6 \%)$ & 0 & $3(13.6 \%)$ & 0 & $16(72 \%)$ & 0 & 0 & 0 & 0 \\
\hline Appache (34) & 0 & 0 & 0 & $2(6 \%)$ & $32(94 \%)$ & 0 & & 0 & 0 & 0 \\
\hline $\begin{array}{l}\text { Mailbomb } \\
\text { (26) }\end{array}$ & 0 & $26(100 \%)$ & 0 & 0 & 0 & 0 & 0 & 0 & 0 & 0 \\
\hline Snmpget(8) & 0 & $8(100 \%)$ & 0 & & 0 & 0 & 0 & 0 & 0 & 0 \\
\hline
\end{tabular}

Citation: Fasidi FO, Adebayo OT. Detecting Distributed Denial-of-Service DDoS Attacks. Curr Tr Comp Sci \& App 1(2)- 2019. CTCSA. 
Table 28: Confusion matrix obtained from one and two attribute combination from test dataset (unprune rules).

\begin{tabular}{|c|c|c|c|c|c|c|c|c|c|c|}
\hline & Pod & Smurf & Teardrop & Neptune & Land & Normal & Appache & Mail bomb & Snmpget & Unknown \\
\hline Pod (40) & 0 & 0 & 0 & 0 & $40(10 \%)$ & 0 & 0 & 0 & 0 & 0 \\
\hline Smurf (107) & 0 & $107(100 \%)$ & 0 & 0 & 0 & 0 & 0 & 0 & 0 & 0 \\
\hline Teardrop (9) & 0 & 0 & $7(88 \%)$ & 0 & $2(12 \%)$ & 0 & 0 & 0 & 0 & 0 \\
\hline Neptume (44) & 0 & 0 & 0 & 0 & $44(10 \%)$ & 0 & 0 & 0 & 0 & 0 \\
\hline Land (9) & 0 & 0 & 0 & 0 & $9(100 \%)$ & 0 & 0 & 0 & 0 & 0 \\
\hline Normal (22) & 0 & $3(13.6 \%)$ & 0 & $3(13.6 \%)$ & 0 & $16(72 \%)$ & 0 & 0 & 0 & 0 \\
\hline Appache (34) & 0 & 0 & 0 & 0 & $34(10 \%)$ & 0 & 0 & 0 & 0 & 0 \\
\hline Mailbomb (26) & 0 & 0 & 0 & 0 & $26(10 \%)$ & 0 & 0 & 0 & 0 & 0 \\
\hline Snmpget(8) & 0 & $8(100 \%)$ & 0 & & 0 & 0 & 0 & 0 & 0 & 0 \\
\hline
\end{tabular}

Table 29: Confusion matrix obtained from one, two and three attribute combination from test dataset (unprune rules).

\begin{tabular}{|c|c|c|c|c|c|c|c|c|c|c|}
\hline & Pod & Smurf & Teardrop & Neptune & Land & Normal & Appache & Mail bomb & Snmpget & Unknown \\
\hline Pod (40) & 0 & $40(100 \%)$ & 0 & 0 & 0 & 0 & 0 & 0 & 0 & 0 \\
\hline Smurf (107) & 0 & $107(100 \%)$ & 0 & 0 & 0 & 0 & 0 & 0 & 0 & 0 \\
\hline Teardrop (9) & 0 & 0 & $9(100 \%)$ & 0 & 0 & 0 & 0 & 0 & 0 & 0 \\
\hline Neptume (44) & 0 & 0 & 0 & 0 & $44(100 \%)$ & 0 & 0 & 0 & 0 & 0 \\
\hline Land (9) & 0 & 0 & 0 & 0 & $9(100 \%)$ & 0 & 0 & 0 & 0 & 0 \\
\hline Normal (22) & 0 & $2(9 \%)$ & 0 & $3(13.6 \%)$ & 0 & $17(77 \%)$ & 0 & 0 & 0 & 0 \\
\hline Appache (34) & 0 & 0 & 0 & 0 & $34(10 \%)$ & 0 & 0 & 0 & 0 & 0 \\
\hline Mailbomb (26) & 0 & 0 & 0 & 0 & $26(10 \%)$ & 0 & 0 & 0 & 0 & 0 \\
\hline Snmpget(8) & 0 & $8(100 \%)$ & 0 & & 0 & 0 & 0 & 0 & 0 & 0 \\
\hline
\end{tabular}

Table 30: Confusion matrix obtained from one, two, three and four attribute combination from test dataset (unprune rules).

\begin{tabular}{|c|c|c|c|c|c|c|c|c|c|c|}
\hline & Pod & Smurf & Teardrop & Neptune & Land & Normal & Appache & Mail bomb & Snmpget & Unknown \\
\hline Pod (40) & 0 & $40(10 \%)$ & 0 & 0 & 0 & 0 & 0 & 0 & 0 & 0 \\
\hline Smurf (107) & 0 & $107(100 \%)$ & 0 & 0 & 0 & 0 & 0 & 0 & 0 & 0 \\
\hline Teardrop (9) & 0 & 0 & $9(100 \%)$ & 0 & 0 & 0 & 0 & 0 & 0 & 0 \\
\hline Neptume (44) & 0 & 0 & 0 & $10(22.7 \%)$ & $34(77.8 \%)$ & 0 & 0 & 0 & 0 & 0 \\
\hline Land (9) & 0 & 0 & 0 & 0 & $9(100 \%)$ & 0 & 0 & 0 & 0 & 0 \\
\hline Normal (22) & 0 & $2(9)$ & 0 & $3(13.6 \%)$ & 0 & $17(77 \%)$ & 0 & 0 & 0 & 0 \\
\hline Appache (34) & 0 & 0 & 0 & 0 & $34(100 \%)$ & 0 & 0 & 0 & 0 & 0 \\
\hline Mailbomb (26) & 0 & 0 & 0 & 0 & $26(100 \%)$ & 0 & 0 & 0 & 0 & 0 \\
\hline Snmpget(8) & 0 & 0 & $8(100 \%)$ & & 0 & 0 & 0 & 0 & 0 & 0 \\
\hline
\end{tabular}

Table 31: Confusion matrix obtained from one, two, three, four and five attribute combination from test dataset (unprune rules).

\begin{tabular}{|c|c|c|c|c|c|c|c|c|c|c|}
\hline & Pod & Smurf & Teardrop & Neptune & Land & Normal & Appache & Mail bomb & Snmpget & Unknown \\
\hline Pod (40) & $40(100 \%)$ & 0 & 0 & 0 & 0 & 0 & 0 & 0 & 0 & 0 \\
\hline Smurf (107) & 0 & $107(100 \%)$ & 0 & 0 & 0 & 0 & 0 & 0 & 0 & 0 \\
\hline Teardrop (9) & 0 & 0 & $9(100 \%)$ & 0 & 0 & 0 & 0 & 0 & 0 & 0 \\
\hline $\begin{array}{l}\text { Neptume } \\
(44)\end{array}$ & 0 & 0 & 0 & $10(22.7 \%)$ & $34(77.8 \%)$ & 0 & 0 & 0 & 0 & 0 \\
\hline Land (9) & 0 & 0 & 0 & 0 & $9(100 \%)$ & 0 & 0 & 0 & 0 & 0 \\
\hline Normal (22) & 0 & $2(9 \%)$ & 0 & $3(13.6 \%)$ & 0 & $17(77 \%)$ & 0 & 0 & 0 & 0 \\
\hline Appache (34) & 0 & 0 & 0 & 0 & $34(100 \%)$ & 0 & 0 & 0 & 0 & 0 \\
\hline $\begin{array}{l}\text { Mailbomb } \\
\text { (26) }\end{array}$ & 0 & 0 & 0 & 0 & $26(100 \%)$ & 0 & 0 & 0 & 0 & 0 \\
\hline Snmpget(8) & 0 & $8(100 \%)$ & 0 & & 0 & 0 & 0 & 0 & 0 & 0 \\
\hline
\end{tabular}


The results in Tables 27-31 were obtained from classification all Land attacks were correctly classified, between $77 \%$ and $90 \%$ of test data set with the raw unpruned rules. From the tables, Pod attacks were classified Teardrop and smurf attacks. Smurf and Teardrop attacks were $100 \%$ and $88 \%$ classified correctly of Normal traffic were classified correctly. $94 \%$ and $6 \%$ of Appache attack were classified as Land and Neptume attack respectively. respectively, all Neptume attacks were classified as Land attacks,

Smnpget attacks were classified as smurf and Teardrop attacks.

Table 32: Confusion matrix obtained from one attribute combination from test dataset (prune rules).

\begin{tabular}{|c|c|c|c|c|c|c|c|c|c|c|}
\hline & Pod & Smurf & Teardrop & Neptune & Land & Normal & Appache & Mail bomb & Snmpget & Unknown \\
\hline Pod (40) & $4(10 \%)$ & 0 & $34(85 \%)$ & 0 & 0 & 0 & 0 & 0 & 0 & $2(5 \%)$ \\
\hline Smurf (107) & 0 & 103(96.3\%) & 0 & 0 & 0 & 0 & 0 & 0 & 0 & $4(3.7 \%)$ \\
\hline Teardrop (9) & 0 & $9(100 \%)$ & 0 & 0 & 0 & 0 & 0 & 0 & 0 & 0 \\
\hline Neptume (44) & $1(2.2 \%)$ & 0 & $3(6.8 \%)$ & $9(20.5 \%)$ & $1(2.2 \%) 5$ & 0 & 0 & $1(2.2 \%)$ & 0 & $29(65.9 \%)$ \\
\hline Land (9) & 0 & 0 & $1(11.1 \%)$ & $1(11.1 \%)$ & $7(77.7 \%)$ & 0 & 0 & 0 & 0 & 0 \\
\hline Normal (22) & 0 & 0 & 0 & $2(9 \%)$ & 0 & $20(91 \%)$ & 0 & 0 & 0 & 0 \\
\hline Appache (34) & 0 & 0 & $1(2.9 \%)$ & 0 & $3(8.82 \%)$ & 0 & & 0 & 0 & $29(85.2 \%)$ \\
\hline Mailbomb (26) & 0 & 0 & $14(53.8 \%)$ & 0 & 0 & 0 & 0 & 0 & 0 & $12(46.1 \%)$ \\
\hline Snmpget(8) & 0 & $8(100 \%)$ & 0 & & 0 & 0 & 0 & 0 & 0 & 0 \\
\hline
\end{tabular}

Table 33: Confusion matrix obtained from one and two attribute combination from test dataset (prune rules).

\begin{tabular}{|c|c|c|c|c|c|c|c|c|c|c|}
\hline & Pod & Smurf & Teardrop & Neptune & Land & Normal & Appache & Mail bomb & Snmpget & Unknown \\
\hline Pod (40) & 0 & $40(100 \%)$ & 0 & 0 & 0 & 0 & 0 & 0 & 0 & 0 \\
\hline Smurf (107) & 0 & $107(100 \%)$ & 0 & 0 & 0 & 0 & 0 & 0 & 0 & 0 \\
\hline Teardrop (9) & 0 & $2(12 \%)$ & $7(88 \%)$ & 0 & 0 & 0 & 0 & 0 & 0 & 0 \\
\hline Neptume (44) & 0 & 0 & 0 & $44(100 \%)$ & 0 & 0 & 0 & 0 & 0 & 0 \\
\hline Land (9) & 0 & 0 & 0 & 0 & $9(100 \%)$ & 0 & 0 & 0 & 0 & 0 \\
\hline Normal (22) & 0 & 0 & 0 & $2(9 \%)$ & 0 & $20(91 \%)$ & 0 & 0 & 0 & 0 \\
\hline Appache (34) & 0 & $1(2.9 \%)$ & 0 & $1(2.9 \%)$ & 0 & 0 & 0 & 0 & 0 & $32(94.1 \%)$ \\
\hline
\end{tabular}

Table 34: Confusion matrix obtained from one, two and three attribute combination from test dataset (prune rules).

\begin{tabular}{|c|c|c|c|c|c|c|c|c|c|c|}
\hline & Pod & Smurf & Teardrop & Neptune & Land & Normal & Appache & Mail bomb & Snmpget & Unknown \\
\hline Pod (40) & $38(95 \%)$ & $2(5 \%)$ & 0 & 0 & 0 & 0 & 0 & 0 & 0 & 0 \\
\hline Smurf (107) & 0 & $107(100 \%)$ & 0 & 0 & 0 & 0 & 0 & 0 & 0 & 0 \\
\hline Teardrop (9) & 0 & 0 & $9(100 \%)$ & 0 & 0 & 0 & 0 & 0 & 0 & 0 \\
\hline Neptume (44) & 0 & 0 & 0 & $44(100 \%)$ & 0 & 0 & 0 & 0 & 0 & 0 \\
\hline Land (9) & 0 & 0 & 0 & 0 & $9(100 \%)$ & 0 & 0 & 0 & 0 & 0 \\
\hline Normal (22) & 0 & 0 & 0 & $2(9 \%)$ & 0 & $20(91 \%)$ & 0 & 0 & 0 & 0 \\
\hline Appache (34) & 0 & $1(2.9 \%)$ & 0 & $1(2.9 \%)$ & 0 & 0 & 0 & 0 & 0 & $32(94.1 \%)$ \\
\hline Mailbomb (26) & 0 & $10(138.5 \%)$ & 0 & 0 & 0 & 0 & 0 & 0 & 0 & $16(61.5 \%)$ \\
\hline Snmpget(8) & 0 & $3(37.5 \%)$ & 0 & & 0 & 0 & 0 & 0 & 0 & $5(62.5 \%)$ \\
\hline
\end{tabular}

Table 35: Confusion matrix obtained from one, two, three and four attribute combination from test dataset (prune rules).

\begin{tabular}{|c|c|c|c|c|c|c|c|c|c|c|}
\hline & Pod & Smurf & Teardrop & Neptune & Land & Normal & Appache & Mail bomb & Snmpget & Unknown \\
\hline Pod (40) & $38(95 \%)$ & $2(5 \%)$ & 0 & 0 & 0 & 0 & 0 & 0 & 0 & 0 \\
\hline Smurf (107) & 0 & $107(100 \%)$ & 0 & 0 & 0 & 0 & 0 & 0 & 0 & 0 \\
\hline Teardrop (9) & 0 & 0 & $9(100 \%)$ & 0 & 0 & 0 & 0 & 0 & 0 & 0 \\
\hline Neptume (44) & 0 & 0 & 0 & $44(100 \%)$ & 0 & 0 & 0 & 0 & 0 & 0 \\
\hline Land (9) & 0 & 0 & 0 & 0 & $9(100 \%)$ & 0 & 0 & 0 & 0 & 0 \\
\hline Normal (22) & 0 & 0 & 0 & $2(9 \%)$ & 0 & $20(91 \%)$ & 0 & 0 & 0 & 0 \\
\hline Appache (34) & 0 & $1(2.9 \%)$ & 0 & $1(2.9 \%)$ & 0 & 0 & 0 & 0 & 0 & $32(94.1 \%)$ \\
\hline Mailbomb (26) & 0 & $10(138.5 \%)$ & 0 & 0 & 0 & 0 & 0 & 0 & 0 & $16(61.5 \%)$ \\
\hline Snmpget(8) & 0 & $3(37.5 \%)$ & 0 & & 0 & 0 & 0 & 0 & 0 & $5(62.5 \%)$ \\
\hline
\end{tabular}


Table 36: Confusion matrix obtained from one, two, three, four and five attribute combination from test dataset (prune rules).

\begin{tabular}{|c|c|c|c|c|c|c|c|c|c|c|}
\hline & Pod & Smurf & Teardrop & Neptune & Land & Normal & Appache & Mail bomb & Snmpget & Unknown \\
\hline Pod (40) & $38(95 \%)$ & $2(5 \%)$ & 0 & 0 & 0 & 0 & 0 & 0 & 0 & 0 \\
\hline Smurf (107) & 0 & $107(100 \%)$ & 0 & 0 & 0 & 0 & 0 & 0 & 0 & 0 \\
\hline Teardrop (9) & 0 & 0 & $9(100 \%)$ & 0 & 0 & 0 & 0 & 0 & 0 & 0 \\
\hline Neptume (44) & 0 & 0 & 0 & $14(100 \%)$ & 0 & 0 & 0 & 0 & 0 & 0 \\
\hline Land (9) & 0 & 0 & 0 & 0 & $9(100 \%)$ & 0 & 0 & 0 & 0 & 0 \\
\hline Normal (22) & 0 & 0 & 0 & $2(9 \%)$ & 0 & $20(91 \%)$ & 0 & 0 & 0 & 0 \\
\hline Appache (34) & 0 & $1(2.9 \%)$ & 0 & $1(2.9 \%)$ & 0 & 0 & 0 & 0 & 0 & $32(94.1 \%)$ \\
\hline Mailbomb (26) & 0 & $10(138.5 \%)$ & 0 & 0 & 0 & 0 & 0 & 0 & 0 & $16(61.5 \%)$ \\
\hline Snmpget(8) & 0 & $3(37.5 \%)$ & 0 & & 0 & 0 & 0 & 0 & 0 & $5(62.5 \%)$ \\
\hline
\end{tabular}

The results in Tables 32-36 were obtained from classification of test data set with the raw pruned rules. 3,4, and 5 attributes rules classified Pod, Smurf Teardrop, Neptume and Land attacks correctly. Appache, mailbomb and snmpget attacks were classified as either Unknown, Smurf or Teardrop attacks. Table 37 shows

the summary of all the correctly classified attacks. All the attacks present in the test dataset which were not used for training of the Association rule classifier were classified as attacks with unprune rule, the pruned rule classified these attacks as unknown attacks. Tables 38 \& 39 below shows how they were classified [21-46].

Table 37: Summary Correctly Classsified Attacks from the Test Dataset.

\begin{tabular}{|c|c|c|c|c|c|c|c|c|c|}
\hline & Pod & Smurf & Teardrop & Neptune & Land & Normal & Appache & Mail bomb & Snmpget \\
\hline Table 32 & 0 & $96 \%$ & 0 & $22.50 \%$ & $77.70 \%$ & $91 \%$ & 0 & 0 & 0 \\
\hline Table 33 & 0 & $100 \%$ & $88 \%$ & $100 \%$ & $100 \%$ & $91 \%$ & 0 & 0 & 0 \\
\hline Table 34 & $95 \%$ & $100 \%$ & $100 \%$ & $100 \%$ & $100 \%$ & $91 \%$ & 0 & 0 & 0 \\
\hline Table 35 & $95 \%$ & $100 \%$ & $100 \%$ & $100 \%$ & $100 \%$ & $91 \%$ & 0 & 0 & 0 \\
\hline Table 36 & $95 \%$ & $100 \%$ & $100 \%$ & $100 \%$ & $100 \%$ & $91 \%$ & 0 & 0 & 0 \\
\hline
\end{tabular}

Table 38: Classification of Attacks not Present in the Test Data (unprune Rule).

\begin{tabular}{|c|c|c|c|c|c|}
\hline & Pod & Smurf & Teardrop & Neptune & Land \\
\hline Appache & & & $1(2.9 \%)$ & $1(2.9 \%)$ & $34((100 \%)$ \\
\hline mailbomb & & & & & $26(100 \%)$ \\
\hline Snmpget & & & $8(100 \%)$ & & \\
\hline
\end{tabular}

Table 39: Classification of Attacks not Present in the Test Data (prune Rule).

\begin{tabular}{|c|c|c|c|c|c|c|}
\hline & Pod & Smurf & Teardrop & Neptune & Land & Unknown \\
\hline Appache & & $2(6 \%)$ & & & $32(94.1 \%)$ \\
\hline mailbomb & & $10(38.5 \%)$ & & & $16(61.5 \%)$ \\
\hline Snmpget & & $3(62.5 \%)$ & & & $5(62.5 \%)$ \\
\hline
\end{tabular}

\section{Conclusion}

The need for effective and efficient security on our system cannot be over-emphasized. This position is strengthened by the degree of human dependency on computer systems and the electronic superhighway (Internet) which grows in size and complexity on daily basis for business transactions, source of information or research. Association Rule methods of improving intrusion detection systems based on machine learning techniques were described and implemented on Intel Duo-core, CPU 2.88GHz, 1024MB RAM using Java programming language.

The work is motivated by increasing growth in importance of intrusion detection to the emerging information society. The research work provided background detail on intrusion detection techniques, and briefly described intrusion detection systems. In this research, an Association rule-based algorithm, was newly developed for mining known known-patterns. The results of the developed tools are satisfactory though it can be improved upon. These tools will go a long way in alleviating the problems of security of data by detecting security breaches on computer system.

\section{References}

1. Brignoli, Delio (2008) DDoS detection based on traffic self-similarity. University of Canterbury. Computer Science and Software Engineering.

2. Yeonhee Lee, Youngseok Lee (2011) Detecting DDoS Attacks with Hadoop. 
3. Adetunmbi AO, Zhiwei Shi, Zhongzhi Shi, Adewale Olumide S (2006) Network Anomalous Intrusion Detection using Fuzzy-Bayes. IFIP International Federation for Information Processing, Intelligent Information Processing III, (Boston: Springer) 228: 525 -530.

4. Agrawal R, Sikrant R (1994) Fast Algorithms for mining association rules. In Proceedings of the $20^{\text {th }}$ VLDB Conference, Santiago, Chile.

5. Aha D (1997) Lazy learning. In Artificial Intelligence. Kluwer Academic Publishers 11: 7-10.

6. Aha D, Kibler D, Albert M (1991) Instance-based learning algorithms. Machine Learning 6(1): 37-66.

7. Anderson D, Frivoid T, Valdes A (1995) Detecting Unusual Program Behaviour using the Statistical Component of the Next-generation Intrusion Detection Expert System (NIDES). Computer Science Laboratory SRI-CSL 95-06.

8. Bellovin SM (1993) Packets Found on an Internet. Computer Communications Review 23(3): 26-31.

9. Beverly R (2003) MS-SQL Slammer/Sapphire Traffic Analysis. MIT LCS.

10. Cohen WW (1995) Fast Effective Rule Induction. In Machine Learning: the $12^{\text {th }}$ International Conference, Lake Tahoe, CA.

11. Cohen W (1995) Fast effective rule induction. In Machine Learning Proceedings of the Twelfth International Conference.

12. Daelemans W, Van den Bosch, A Zavrel J (1999) Forgetting exceptions is harmful in language learning. In Machine Learning 34(1-3): 11-41.

13. Daelemans W, Zavrel J, Van Der Sloot K, Van Den Bosch A (2005) TiMBL: Tilburg Memory-Based Learning - Reference Guide. Induction of Linguistic Knowledge.

14. Deraison R (2003) Nessus.

15. Farmer D Venema W (1993) Improving the Security of Your Site by Breaking Into it.

16. Fayyad (1996a) Mining Scientific Data. Communications of the ACM 39(11): 51-57.

17. Fayyad (1996b) From Data Mining to Knowledge Discovery: An Overview. In Advances in Knowledge Discovery and Data Mining. Fayyad U, Piatesky-Shappiro G, Smyth P, Uthurusamy R (Eds.). AAAI/MIT Press, Cambridge, MA

18. Fayyad, Gregory Piatetsky Shapiro and Padhraic Smyth (1996) The KDD Process of Extracting Useful Knowledge from Volumes of Data. Communications of the ACM 39(11): 27-34.

19. Floyd S, Paxson V (2001) Difficulties in Simulating the Internet. IEEE/ ACM Transactions on Networking.

20. Fyodor (1998) Remote OS detection via TCP/IP Stack Finger Printing.

21. Fyodor (2002) NMAP Idle Scan Technique (Simplified).

22. Fyodor (2003) NMAP.

23. Hall M, Smith L (1996) Practical feature subset selection for machine learning. In Proceedings of the Australian Computer Science Conference (University of Western Australia). University of Waikato.

24. Hendrickx I (2005) Local Classification and Global Estimation. Koninklijke drukkerij Broese \& Peereboom.

25. Ilgun (1995) State Transition Analysis: A Rule-based Intrusion Detection Approach. IEEE Transactions on Software Engineering 21(3): 181-199.
26. Jonsson E, Olovsson T (1997) A Quantitative Model of Security Intrusion Process Based on Atacker Behaviour. IEEE Transactions on Software Engineering 23(4).

27. Kendall K (1999) A Database of Computer Attacks for the Evaluation of Intrusion Detection Systems. Masters Thesis, Massachusetts Institute of Technology.

28. Kumar S, Spafford EH (1995) A Software Architecture to Support Misuse Intrusion Detection. In Proceedings of the $18^{\text {th }}$ National Information Security Conference 1995: 194-204.

29. Lunt (1992) A Real-time Intrusion Detection Expert System (IDES). A Final Technical Report Technical Report. Computer science Laboratory, SRI International, Menlo Park, California, February 1992.

30. Lunt T (1993) Detecting Intruders in Computer Systems. In Proceedings of the 1993 Conference on Auditing and Computer Technology 1993.

31. Mitchell T (1997a) Does machine learning really work? In AI Magazine, p. 11-20.

32. Mitchell T (1997b) Machine Learning. McGraw-Hill International Editions.

33. Moore Paxson V, Savage S, Shannon C, Staniford S, Weaver N (2003) The Spread of the Sapphire/Slammer Worm. CAIDA, ICSI, Silicone Defense, UC Berkeley EECS and UC San Diego CSE.

34. Pietraszek T (2004) Using adaptive alert classification to reduce false positives in intrusion detection. In Recent Advances in Intrusion Detection 3224: 102-124.

35. Sanfilippo S (2003) HPING.

36. SANS (2000) An Analysis of the "Shaft" Distributed Denial of Service Tool. SANS Institute.

37. Spafford H (1998) The Internet Worm Program: An Analysis. Purdue Technical Report CSDTR-823.

38. Standler Ronald B (2001) Computer Crime.

39. Staniford S, Paxson V, Weaver N (2002) How to Own the Internet in your Spare Time. Proc. $11^{\text {th }}$ USENIX Security Symposium.

40. Van Den Bosch A, Daelemans W (1998) Do not forget full memory in memory-based learning of word pronunciation. In NeMLaP3/CoNLL 98: 195-204.

41. Van Den Bosch A, Krahmer E, Swerts M (2001) Detecting problematic turns in human machine interactions: Rule-induction versus memorybased learning approaches. In Meeting of the Association for Computational Linguistics ( $\left.2^{\text {nd }} e d n\right) .$, pp. 499-506.

42. Weiser Kevin (2002) Valve Launches Largest DDoS in History.

43. Wang Y, Wang, J, Miner A (2004) Anomaly intrusion detection system using one class SVM. 5th Annual IEEE Information Assurance Workshop, West Point, New York.

44. Warren RH, Johnson JA, Huang GH (2004) Application of Rough Sets to Environmental Engineering Models. JF Peters (Eds.).: Transaction on Rough Sets. LNCS Springer verlag Berlin Heidelberg 3100: 356-374.

45. William W Streilein, David J Fried, Robert K Cunningham (2005) Detecting Flood-based Denial-of-Service Attacks with SNMP/RMON.

46. Van Ryan, Jane S (1998) Center for Information Security Technolog Releases CMDS Version. 
(C) (1) This work is licensed under Creative Commons Attribution 4.0 License

To Submit Your Article Click Here: Submit Article

DOI: $10.32474 /$ CTCSA.2019.01.000110

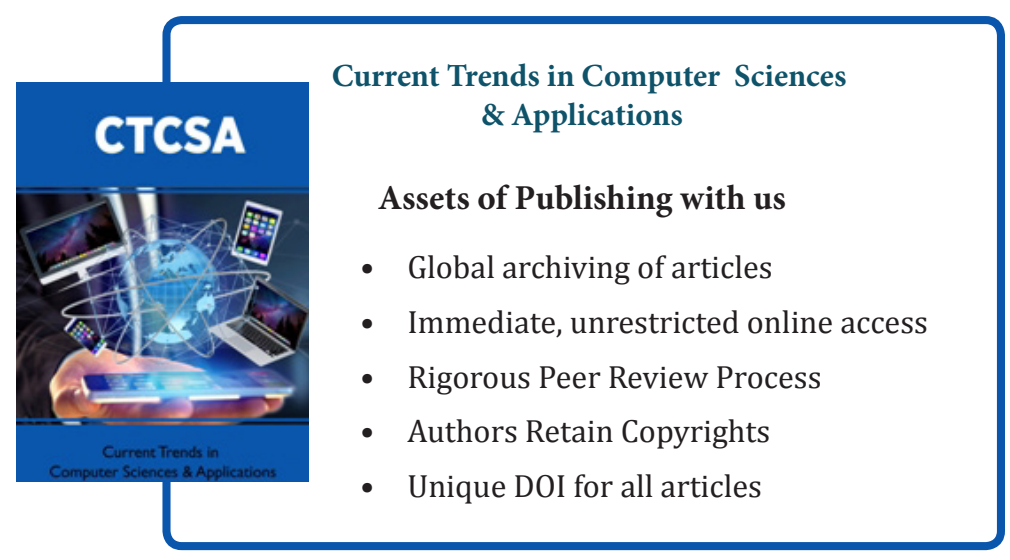

